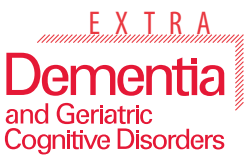

\title{
Responding to Varying Levels of Certainty about Pain in People with Dementia after Initial Pain Assessment
}

\author{
Mohammad Rababa Mohammed Aldalaykeh \\ Jordan University of Science and Technology, Irbid, Jordan
}

\author{
Keywords \\ Nurses' certainty · Pain assessment - People with dementia - Agitation · Nursing homes . \\ Dementia
}

\begin{abstract}
Introduction: Pain is still inadequately treated in people with dementia (PWD) due to the complexity of assessing it. Pain assessment in PWD is still challenging because of patientrelated or nurse-related factors. The patient-related factors have been studied extensively. However, the nurse-related factors, e.g., nurses' certainty regarding suspected pain in PWD, have been given little attention by researchers. Purpose: This study aims to investigate how the degree of nurses' certainty is compromised after pain assessment and how this affects levels of pain and agitation in PWD. Method: A descriptive, correlational design was used, and a convenience sample of 104 nursing home residents with dementia were recruited. Results: This study found that nurses' certainty after the pain assessment mediates the relationship between the type of pain assessment and patient outcomes when the nurses had a high initial certainty. When nurses had a low initial certainty, their certainty after the assessment and the type of assessment predicted the levels of pain and agitation in PWD. Conclusion: Understanding how nurses' certainty can be compromising after pain assessment and how this relates to pain management in PWD is crucial.

\section{Introduction}

Pain is a very prevalent problem among older adults, but it is even more prevalent among those with dementia [1]. Fifty to eighty percent of people with dementia (PWD) residing in nursing homes (NHs) in the USA only suffer from pain [2]. The high prevalence of unrelieved 
Rababa and Aldalaykeh: Responding to Varying Levels of Certainty about Pain in

pain in PWD has also been consistently confirmed and documented in several studies [3-6]. For instance, a recent study [7] has reported that, even with controlling for the number of painful situations, for older adults with dementia, significantly fewer analgesics are prescribed and received than for those without dementia. According to a recent study, 47\% of PWD residing in NHs report pain daily, but 33\% of these do not receive any analgesics at all [6].

Unrelieved pain in PWD is associated with many negative health consequences including agitation, physical disability, poor appetite, aggression, social isolation, depression, sleep and activity imbalances, functional and cognitive impairment, a poor quality of life, prolonged institutionalization [6], nutritional deficiencies, impaired mobility, reduced immune function, and high mortality [8].

Reasons for unrelieved pain in PWD include: (a) the complexity of the pain assessment (b) no treatment or undertreatment of pain, and (c) factors relating to nurses' decisionmaking skills. To ensure effective and timely management of pain, nurses should select the most effective and accurate method of assessment [9]. More critical ways could also be developed to effectively and appropriately assess pain in PWD.

Poor pain assessment in PWD is also related to the effects of cognitive impairment and the dementia process. Cognitive impairment in PWD makes them unable to clearly and meaningfully verbalize their pain and communicate with nurses about their unmet comfort needs [10]. In addition, the dementia process compromises the affective aspect of pain in PWD, complicating their pain arousal or expectation [11]. Moreover, behavioral changes associated with pain are inconsistent among PWD, impeding the ability of nurses to understand and interpret these changes. Therefore, nurses may be reluctant or unwilling to perform a thorough assessment based on an analytical thought process. Instead, they may over-simplify the assessment and not go through an evidence-based decision-making process [12]. They may also perform several treatment trials before administering analgesics, e.g., start with nonpharmacological treatment or antipsychotic drugs to address pain in PWD and delay analgesics.

Poor pain assessment and the ineffective treatment of pain in PWD related to the lack of assessment tools and the disease process of dementia have been extensively studied. Little is known about nurses' decision-making process related to pain assessment and treatment in PWD. The problem of unrelieved pain in PWD may be partially explained by poor decisionmaking [13]. Nurses undertake what is frequently a compromising nonlinear decision-making process before analgesics can be administered to PWD [13]. They may also apply a trial-anderror approach when dealing with episodes of pain experienced by PWD. This can lead to confusion and uncertainty about the pain experienced by PWD [13].

\section{Theoretical Framework}

Only 1 theoretical model has directly discussed the concept of nurses' certainty and how it relates to the problem of inadequate treatment of pain in PWD. The Response to Certainty of Pain (RCP) model [13] illuminates 2 trajectories of pain assessment and treatment in PWD. The model provides an explanation of how the level of nurses' certainty may predict the level of pain and agitation in $\mathrm{NH}$ residents with dementia. It suggests that when nurses are certain about pain in PWD, they just need to complete a brief self-report assessment and then administer an analgesic in a timely manner to treat pain and agitation. The model proposes that when there is certainty, there is no need for a deep analytical thought process, and that conducting a brief self-report assessment of pain is sufficient. When nurses are not certain about pain, they must conduct additional assessment (multidimensional), and so the treatment of pain and agitation may be delayed. This additional assessment is needed to facilitate the 
Rababa and Aldalaykeh: Responding to Varying Levels of Certainty about Pain in People with Dementia after Initial Pain Assessment

nurses' use of a deeper analytical thought process. Another tenet of the RCP model is that nurses' over-reliance on a brief self-report of pain in PWD if there is uncertainty actually contributes to the uncertainty and to poor outcomes. An additional (multidimensional) assessment may or may not utilize a self-report, but also involves assessing for behavioral and functional changes [13].

A recent study [12] empirically tested the RCP model and all hypotheses deduced from the model were supported in the study. However, no study has yet examined how nurses' certainty after assessment is compromised and how this affects the relationship between the type of pain assessment used and the levels of pain and agitation in PWD. According to the RCP model, nurses' certainty after conducting initial pain assessment mediates the relationship between the type of pain assessment employed and the levels of pain and agitation in PWD. The purpose of this study was to examine this mediating effect.

The hypothesis deduced from the RCP model when nurses have high initial certainty is:

1 The relationship between the type of pain assessment and the levels of pain and agitation in PWD will be mediated by nurses' certainty after the assessment.

Rationale: According to the RCP model, when nurses' certainty is not compromised, the PWD in their care will have better pain and agitation outcomes than the PWD cared for by nurses whose certainty is compromised after conducting a brief assessment.

The hypothesis deduced from the RCP model when nurses have low initial certainty is:

2 The type of pain assessment and nurses' certainty after the assessment will predict the levels of pain and agitation in PWD.

Rationale: According to the RCP model, when nurses' certainty is compromised, the PWD in their care will have better pain and agitation outcomes if they receive an additional assessment than the PWD cared for by nurses who conduct a brief assessment.

\section{Method}

\section{Design, Setting, and Sample}

The researchers used a correlational descriptive study design. The study was conducted in $4 \mathrm{NHs}$ in Jordan. A convenience sample of $104 \mathrm{NH}$ residents with dementia were recruited. The sample size was calculated after doing $G^{*}$ power analysis using an a priori calculator of sample size for multiple regression. Given an anticipated medium effect size of 0.15 , a $p$ value of $0.05,2$ predictors, and a level of power of 0.95 , the minimum required sample size was 104 . The eligible participants were any NH resident who: (1) had any degree of cognitive impairment noticed by family, reported by nurses, or documented in their medical records, (2) suffered from any disease-causing pain like musculoskeletal disease, cancer, or urinary tract infections, and (3) complained of any behavioral or functional changes associated with pain; examples of behavioral changes included (but were not limited to) crying or screaming for no obvious reason, frequent rubbing of body parts, and odd or abnormal postural positions; examples of functional changes included (but were not limited to) changes in appetite or sleep pattern, and (4) were cared for by nurses who had been working in the $\mathrm{NH}$ setting for at least 2 years.

\section{Measurement}

Pain

Pain was measured by the Pain Assessment in Advanced Dementia (PAINAD) scale [14]. The PAINAD tool has 5 items including breathing, negative vocalization, facial expression, body language, and consolability. Each item scores 0 , 1, or 2, with the total score ranging from 0 to 10 to indicate pain severity $(0=$ no pain and $10=$ severe pain). The PAINAD tool has 
Rababa and Aldalaykeh: Responding to Varying Levels of Certainty about Pain in

acceptable reliability and internal consistency. This tool was applied twice for each resident: during a rest period and during caregiving.

Agitation

Agitation was measured by the Wisconsin Agitation Inventory (WAI) [15], with a total score ranging from 0 to 100 . The WAI is an observational scale that measures agitation by directly observing agitated behaviors. These include arguing, irritability, aggression, verbal threats, and destroying property. The tool was applied 4 times for each resident: at breakfast, mid-morning, before dinner, and after dinner.

Certainty after Assessment

Upon review of the literature, no standard tool that specifically measures nurses' certainty of pain was found. This was thus measured by a 1-item Likert scale with multiple options: $1=$ very uncertain, $2=$ uncertain, $3=$ don't know, $4=$ certain, and $5=$ very certain. This is a popular means for measuring attitudes, positions, and decisions. The scale enables the observer to operationalize the concept using the best method available to capture the conceptual definition of certainty, i.e., a self-report. The nurses indicated their own attitude, position, and decision about suspected pain in PWD along the scale.

Type of Pain Assessment

Data about the type of pain experienced by PWD was collected directly from their medical records and coded based on the following rubric: 1, a brief self-report (e.g., pain or no pain); 2 , a numerical scale (0-10); 3 , a face scale; 4 , a behavioral assessment (behavioral signs of pain); 5, a functional assessment (i.e., changes in appetite or sleep pattern). Kovach et al. [16] used this coding rubric of pain assessment in PWD successfully. Scores of 1-3 were considered as a brief assessment and scores of 4 and 5 as additional assessment. Interrater reliability checks were done for every $10 \%$ of data collection to prevent any measurement error. The interrater reliability was very good $(0.84)$.

\section{Dementia}

The level of residents' dementia was measured using the Arabic versions of the Mini-Cog test and the revised Mini-Mental State Examination (MMSE-2). A recent study [17] recommended using MMSE-2 and Mini-Cog together to increase the sensitivity and specificity of the MMSE-2 that is otherwise limited the by the confounding effects of cultural bias, language, and level of education. The Mini-Cog test is very easy to administer, brief, and accessible to patients [18]. It has a simple scoring and a high sensitivity with no limitation based on a patient's education, language, and culture. It includes a clock-drawing test and a non-cued recall of 3 unrelated words. The total score is 4 ; a score of $\leq 2$ means there is dementia and a score $>2$ means there is no dementia. The MMSE- 2 is the revised version of the original MMSE [19]. It has the same structure and scoring system as the MMSE with replacement and modification of some items that are problematic for translation into Arabic.

\section{Procedure}

The researcher discussed the eligibility criteria with the $\mathrm{NH}$ administrators and nurses to obtain the initial list of potential participants. The researcher administered the Mini-Cog first to confirm the presence of dementia and determine which participants were eligible, and then the MMSE-2 to measure the level of dementia for these participants. The researcher asked the nurses to start assessing pain and also document the type of pain assessment used when they noticed changes in their patients' behavior or function believed to be associated with pain. Based on the type of pain assessment used, the nurses rated and recorded the level 
Table 1. Hierarchical regression analysis of pain time 1 scores

\begin{tabular}{llrrrr}
\hline Step and predictor variable & $r^{2}$ & $\Delta r^{2}$ & $\beta$ & $t$ & $p$ \\
\hline $\begin{array}{l}\text { Step } 1 \\
\quad \text { Type of pain assessment }\end{array}$ & 0.306 & $0.306^{*}$ & 0.585 & 3.06 & $0.007^{*}$ \\
$\quad \begin{array}{l}\text { Step 2 } \\
\quad \text { Type of pain assessment }\end{array}$ & 0.559 & $0.253^{*}$ & 0.362 & 2.205 & 0.061 \\
$\quad$ Nurses' certainty & & & -0.567 & -3.46 & $0.003^{*}$ \\
$F_{2,23}=13.54, p<0.0001$ & & & & & \\
\hline$* p<0.05$.
\end{tabular}

Table 2. Hierarchical regression analysis of pain time 2 scores

\begin{tabular}{|c|c|c|c|c|c|}
\hline Step and predictor variable & $r^{2}$ & $\Delta r^{2}$ & $\beta$ & $t$ & $p$ \\
\hline \multicolumn{6}{|l|}{ Step 1} \\
\hline Type of pain assessment & 0.144 & $0.144^{*}$ & 0.435 & 2.05 & $0.054^{*}$ \\
\hline Step 2 & & & & & \\
\hline Type of pain assessment & 0.547 & $0.403^{*}$ & 0.150 & 0.932 & 0.364 \\
\hline $\begin{array}{c}\text { Nurses' certainty } \\
F_{2,23}=14.477, p<0.0001\end{array}$ & & & -0.723 & -4.501 & $0.001^{*}$ \\
\hline
\end{tabular}

of their certainty regarding the suspected pain. Based on a recommendation by Kovach (pers. commun., 2018), who has extensively measured both outcomes, the researcher returned a week later to measure the levels of patients' pain and agitation. The measurement of pain was done twice, i.e., during rest and caregiving. The measurement of agitation was done 4 times: before breakfast, mid-morning, before dinner, and after dinner.

\section{Data Analysis}

Descriptive statistics were used for the age, length of stay, and level of dementia of the subjects. Hierarchical regression was used to test the mediating effect of nurses' certainty after the assessment on the relationship between the type of pain assessment used and the levels of pain and agitation in PWD. Multiple regression was used to test the second hypothesis.

\section{Description of NH Residents}

The mean age of the residents was 70. 32 (SD 8.45; range 65-85) years and 46.3\% were men. The mean length of stay for the residents was 31.2 (SD 7.33; range 25-73) months. Thirty-four percent $(n=33)$ of the residents had severe dementia; the mean MMSE score was 14.44 and the mean Mini-Cog score was 1.6 (SD 0.54; range 0-2). Nurses' certainty was compromised after conducting a brief assessment of $21 \%$ of 104 residents and after conducting an additional assessment of $45 \%$ of 104 residents.

\section{Hypothesis 1}

The hypothesis that nurses' certainty after initial assessment will mediate the relationship between the type of pain assessment and the levels of pain and agitation in PWD when nurses have an initially high level of certainty was supported. It is noteworthy that, at 
Table 3. Hierarchical regression analysis of agitation scores

\begin{tabular}{lccccc}
\hline Step and predictor variable & $r^{2}$ & $\Delta r^{2}$ & $\beta$ & $t$ & $p$ \\
\hline Step 1 & & & & & \\
$\quad$ Type of pain assessment & 0.150 & $0.150^{*}$ & 0.442 & 3.08 & $0.001^{*}$ \\
$\quad$ Step 2 & & & & & \\
$\quad$ Type of pain assessment & 0.620 & $0.470^{*}$ & 0.147 & 0.963 & 0.349 \\
$\quad$ Nurses' certainty & & & -0.740 & -4.86 & $0.001^{*}$ \\
$F_{2,23}=16.744, p<0.0001$ & & & & & \\
\hline$* p<0.05$.
\end{tabular}

Table 4. Multiple regression analysis of agitation scores

\begin{tabular}{lcrrr}
\hline Predictor variable & $r^{2}$ & $\beta$ & $t$ & $p$ \\
\hline Type of pain assessment & & 0.162 & 2.492 & $0.013^{*}$ \\
Nurses' certainty & 0.129 & $-0.502^{*}$ & -5.963 & $<0.001^{*}$ \\
$F_{2,75}=22.04, p<0.0001$ & & & \\
\hline$* p<0.05$. & & & \\
\hline
\end{tabular}

Step 1, the type of pain assessment accounted for a large percentage of variance in agitation, pain time 1, and pain time 2 outcomes (30.6, 14.4, and 15\%, respectively). However, as seen in Tables $1-3$, the $\beta$ value for the type of pain assessment changed from "significant" in Step 1 to "not significant" in Step 2 when nurses' certainty was added to the regression model.

\section{Hypothesis 2}

A multiple linear regression was calculated to predict patients' outcomes based on the type of pain assessment and nurses' certainty after the initial assessment when nurses had an initially low level of certainty of suspected pain. As seen in Tables 4-6, a significant regression equation was found for pain time $1\left(F_{2,75}=17.14, p<0.0001, R^{2}=0.166\right)$, pain time $2\left(F_{2,75}=14.5317 .14, p<0.0001, R^{2}=0.126\right)$, and agitation $\left(F_{2,75}=22.04, p<0.0001\right.$, $\left.R^{2}=0.129\right)$. Nurses' certainty was statistically significant while the $\beta$ value for the type of pain assessment was insignificant for pain time $2(p=0.304)$ and pain time $1(p=0.270)$. This result indicates that differences in the level of pain during rest and during caregiving in PWD were mainly dependent on nurses' certainty.

\section{Discussion}

This study found that, if nurses' certainty was compromised, i.e., if it went from high to low after the initial pain assessment, this mediated the relationship between the type of pain assessment and the levels of pain and agitation in PWD. In other words, regardless of the type of pain assessment used, the pain and agitation outcomes were worse in PWD when the nurses' certainty was compromised after the initial assessment than the outcomes in PWD cared for by nurses who maintained a high level of certainty. This finding is consistent with the RCP model [13]. When the nurses' level of certainty changes from certain to uncertain 
Rababa and Aldalaykeh: Responding to Varying Levels of Certainty about Pain in People with Dementia after Initial Pain Assessment

Table 5. Multiple regression analysis of pain time 1 scores

\begin{tabular}{lcccc}
\hline Predictor variable & $r^{2}$ & $\beta$ & $t$ & $p$ \\
\hline Type of pain assessment & 0.166 & 0.032 & 0.704 & 0.270 \\
Nurses' certainty & & $-0.410^{*}$ & -4.72 & $<0.001^{*}$ \\
$F_{2,75}=17.14, p<0.0001$ & & & \\
\hline$* p<0.05$ & & & \\
\hline
\end{tabular}

Table 6. Multiple regression analysis of pain time 2 scores

\begin{tabular}{lcccc}
\hline Predictor variable & $r^{2}$ & $\beta$ & $t$ & $p$ \\
\hline Type of pain assessment & 0.126 & 0.060 & 0.641 & 0.304 \\
Nurses' certainty & & $-0.379 *$ & -4.266 & $<0.001^{*}$ \\
$F_{2,75}=14.53, p<0.0001$ & & & \\
\hline$* p<0.05$. & & & & \\
\hline
\end{tabular}

after conducting the initial assessment, analgesics will be delayed, underprescribed, or not administered at all, and therefore pain and agitation in PWD get worse. A descriptive, correlational study [12] was the first study that empirically tested the concept of nurses' certainty and its relation to the issue of inadequate treatment of pain in PWD. However, that particular study did not examine how nurses' level of certainty changed after conducting pain assessment in PWD and how this change is reflected in patient outcomes. No other study has examined how nurses' certainty is compromised after the initial pain assessment, but a recent study [20] has examined how nurses' level of confidence about the presence of pain changes after the pain assessment in PWD. Nurses' confidence about the presence of pain is believed to be interchangeable with nurses' certainty regarding suspected pain. According to the above study [20], nurses who conducted a pain assessment in PWD without validating their confidence about the presence of pain did not significantly decrease the level of discomfort in PWD. This finding emphasizes the importance of nurses' certainty in decision-making regarding pain treatment in PWD.

This study found that when nurses initially have low certainty, the type of pain assessment and nurses' certainty after the initial assessment are significant predictors of the levels of pain and agitation in PWD. According to the RCP model, when nurses have low initial certainty, they may compromise their certainty regarding suspected pain if they do not conduct more detailed or additional assessment. Instead, they may refer to their intuition about the subjective and objective pain data. Therefore, nurses' ability to make certain decisions about pain treatment is limited. They can remain stuck in a trial-and-error approach to pain management and may not provide analgesics at all, which can result in worse pain and agitation outcomes in PWD. On the other hand, if nurses with initially low certainty conduct additional assessment, they may be able to make alternative decisions about pain treatment and provide analgesics [13]. As a result, PWD would have better pain and agitation outcomes. This finding is consistent with previous studies [19,21-23].

According to a recent study [21], sometimes nurses cannot discriminate between pain and dementia. They acknowledge that they are uncertain whether their patients' behavioral 
Rababa and Aldalaykeh: Responding to Varying Levels of Certainty about Pain in

People with Dementia after Initial Pain Assessment

changes are dementia-related or pain-related. Consequently, nurses who are unable to change their doubts would try to manage these changes in behavior according to a pragmatic approach rather than theoretical considerations. As a nurse often has no definitive diagnosis of pain in PWD, they struggle with treatment options and frequently question their decisions about the treatment chosen. They tend to be reluctant and unwilling to provide analgesics and more comfortable with providing antipsychotic medications or else just look out for a reversal of behavioral changes [19]. The use of antipsychotic drugs is often associated with functional disabilities, unmet comfort needs, an increased risk of cardiovascular problems, and a decline in quality of life [22]. In addition, when nurses have a low level of certainty regarding pain in PWD, they look for other relevant information and seek more assessment of pain. Understanding other relevant information includes recognizing comorbid problems, nurses' personal perception of pain, and pain cues specific for PWD [23]. When nurses are able to understand all the relevant information and conduct more detailed assessments, they can confirm when pain is present and provide analgesics [23].

\section{Limitations}

This study has some limitations related to its descriptive correlational design and the convenient sampling method. Using self-report questionnaires for data collection may also have increased the probability of recall bias, which could affect the reliability of the data and influence the results. In addition, since several PWD were cared for by the same nurse, it may have been difficult for the nurse to clearly recall all the information about each resident. Measures of certainty and resident outcomes were clustered regarding nurses and this clustering was not accounted for in the analyses. It is unknown how this clustering may have influenced the research results. This study may also have suffered from the "Hawthorne effect." The nurses in this study were aware they were participating in a study and changed their behavior. Because they knew their performance was being measured in some way, they may have given inaccurate responses about their certainty of pain.

\section{Future Studies}

Since there is no standard tool available that specifically measures nurses' certainty about pain, a future qualitative research study to develop a measurement tool for nurses' certainty regarding suspected pain in PWD is highly recommended. A correlational study would be useful to examine the association of nurse-related factors including their level of education, years of experience, the presence of PWD in their own households, and job autonomy and their certainty regarding suspected pain in PWD.

\section{Conclusion}

Despite the improvement in the quality of palliative care during the past few decades, unrelieved pain is still a very prevalent problem among PWD. Many research studies have examined the role of patient-related factors on the health outcomes of PWD, e.g., pain and agitation. However, little attention has been given by nursing researchers to nurse-related factors such as nurses' certainty. It is important to investigate how nurses' certainty can become compromised after a pain assessment. Given the anticipated increase in the number of PWD that will suffer from pain in the next decades, an improved understanding of nurses' certainty and how it relates to the problem of inadequate treatment of pain in PWD is critical. 
Rababa and Aldalaykeh: Responding to Varying Levels of Certainty about Pain in People with Dementia after Initial Pain Assessment

\section{Statement of Ethics}

Approval was received from the IRB Department of the Jordanian Social Welfare Ministry, which governs all NHs in Jordan. All eligible participants with mild to moderate dementia had their family sign the consent forms. For those participants with severe dementia, power of attorney was used.

\section{Disclosure Statement}

The authors declare that they have no conflicts of interest.

\section{Funding Sources}

This study was funded by the Jordan University of Science and Technology.

\section{Availability of Data and Materials}

The quantitative data used to support the findings of this study are available from the corresponding author upon request.

\section{References}

1 Miu DK, Chan KC. Under-detection of pain in elderly nursing home residents with moderate to severe dementia. J Clin Gerontol Geriatr. 2014;5(1):23-7.

2 Husebo BS, Ballard C, Aarsland D. Pain treatment of agitation in patients with dementia: a systematic review. Int J Geriatr Psychiatry. 2011 Oct;26(10):1012-8.

3 Hunnicutt JN, Ulbricht CM, Tjia J, Lapane KL. Pain and pharmacologic pain management in long-stay nursing home residents. Pain. 2017 Jun; 158(6):1091-9.

4 Mamhidir AG, Sjölund BM, Fläckman B, Wimo A, Sköldunger A, Engström M. Systematic pain assessment in nursing homes: a cluster-randomized trial using mixed-methods approach. BMC Geriatr. 2017 Feb;17(1): 61-87.

5 Monroe TB, Misra SK, Habermann RC, Dietrich MS, Cowan RL, Simmons SF. Pain reports and pain medication treatment in nursing home residents with and without dementia. Geriatr Gerontol Int. 2014 Jul;14(3):541-8.

6 van Kooten J, van der Wouden JC, Sikkes SA, Smalbrugge M, Hertogh CM, Stek ML. Pain neuropsychiatric symptoms and quality of life of nursing home residents with advanced dementia in the Netherlands: a crosssectional study. Alzheimer Dis Assoc Disord. 2017 Oct-Dec;31(4):315-21.

7 Achterberg WP, Pieper MJ, van Dalen-Kok AH, de Waal MW, Husebo BS, Lautenbacher S, et al. Pain management in patients with dementia. Clin Interv Aging. 2013;8:1471-82.

8 van der Maaden T, van der Steen JT, de Vet HC, Hertogh CM, Koopmans RT. Prospective observations of discomfort pain and dyspnea in nursing home residents with dementia and pneumonia. J Am Med Dir Assoc. 2016 Feb;17(2):128-35.

9 Hadjistavropoulos T, Herr K, Prkachin KM, Craig KD, Gibson SJ, Lukas A, et al. Pain assessment in elderly adults with dementia. Lancet Neurol. 2014 Dec;13(12):1216-27.

10 Harrison Dening K. Palliative and end of life care for people with dementia. Nurs Stand. 2016 Feb;30(23):42-9.

11 Kovach CR, editor. Assessing pain and unmet need in patients with advanced dementia: the role of the Serial Trial Intervention (STI). In: Handbook of Pain and Palliative Care. New York: Springer; 2013. pp. 131-44.

12 Rababa M. The association of nurses' assessment and certainty to pain management and outcomes for nursing home residents in Jordan. Geriatr Nurs. 2018 Jan-Feb;39(1):66-71.

13 Gilmore-Bykovskyi AL, Bowers BJ. Understanding nurses' decisions to treat pain in nursing home residents with dementia. Res Gerontol Nurs. 2013 Apr;6(2):127-38.

14 Warden V, Hurley AC, Volicer L. Development and psychometric evaluation of the Pain Assessment in Advanced Dementia (PAINAD) scale. J Am Med Dir Assoc. 2003 Jan-Feb;4(1):9-15.

15 Kovach CR, Taneli Y, Dohearty P, Schlidt AM, Cashin S, Silva-Smith AL. Effect of the BACE intervention on agitation of people with dementia. Gerontologist. 2004 Dec;44(6):797-806.

16 Kovach CR, Hekel B, Rababa M. Feasibility Testing of a Protocol to Stop Ineffective Drug and Nondrug Treatments. West J Nurs Res. 2015 Nov;37(11):1404-22. 
17 Albanna M, Yehya A, Khairi A, Dafeeah E, Elhadi A, Rezgui L, et al. Validation and cultural adaptation of the Arabic versions of the Mini-Mental Status Examination - 2 and Mini-Cog test. Neuropsychiatr Dis Treat. 2017 Mar;13:793-801.

18 Borson S, Scanlan J, Brush M, Vitaliano P, Dokmak A. The mini-cog: a cognitive 'vital signs' measure for dementia screening in multi-lingual elderly. Int J Geriatr Psychiatry. 2000 Nov;15(11):1021-7.

19 Folstein MF, Folstein SE, McHugh PR. "Mini-mental state". A practical method for grading the cognitive state of patients for the clinician. J Psychiatr Res. 1975 Nov;12(3):189-98.

20 Shields CG, Finley MA, Elias CM, Coker CJ, Griggs JJ, Fiscella K, et al. Pain assessment: the roles of physician certainty and curiosity. Health Commun. 2013;28(7):740-6.

21 De Witt Jansen B, Brazil K, Passmore P, Buchanan H, Maxwell D, McIlfactrick SJ, et al. Nurses' experiences of pain management for people with advanced dementia approaching the end of life: a qualitative study. J Clin Nurs. 2017 May;26(9-10):1234-44.

22 Ballard C, Orrell M, Sun Y, Moniz-Cook E, Stafford J, Whitaker R, et al. Impact of antipsychotic review and nonpharmacological intervention on health-related quality of life in people with dementia living in care homes: WHELD-a factorial cluster randomised controlled trial. Int J Geriatr Psychiatry. 2017 Oct;32(10):1094-103.

23 Horgas AL. Pain Management in Older Adults. Nurs Clin North Am. 2017 Dec;52(4):e1-7. 